\title{
Pressure tolerance of Artemia cysts compressed in water medium
}

Rachael Hazael ${ }^{1}$, Shinsuke Matsuda ${ }^{2}$, Yoshihisa Mori², Brianna C.

Fitzmaurice $^{1}$, Gareth Appleby-Thomas ${ }^{1}$, Jonathan D. Painter ${ }^{1}$, Filip

Meersman $^{3}$, Myriam Richaud ${ }^{4}$, Simon Galas ${ }^{4}$, Naurang L. Saini ${ }^{5}$, Fumihisa

$\mathrm{Ono}^{2^{*}}$ and Paul F. McMillan ${ }^{6}$

${ }^{1}$ Centre for Defence Engineering, Cranfield University, The Defence Academy of The United Kingdom, Shrivenham, UK

${ }^{2}$ Department of Applied Science, Okayama University of Science, Okayama, Japan

${ }^{3}$ Department of Chemistry, University of Antwerp, Antwerpen, Belgium

${ }^{4}$ IBMM, University of Montpellier, CNRS, ENSCM, Montpellier, France

${ }^{5}$ Dipartmento di Fisica, Universita di Roma "La Sapienza”, Rome, Italy

${ }^{6}$ Department of Chemistry, University College London, London, UK

Corresponding author: Fumihisa Ono, Department of Applied Science, Okayama

University of Science, 1-1 Ridaicho, Kitaku, Okayama 700-0002, Japan, E-mail:

fumihisaono@yahoo.co.jp 


\section{Anomaly in pressure tolerance of Artemia cysts under high pressure}

The high pressure tolerance of cysts of Artemia salina was investigated up to several GPa in water. No survival was observed after exposure to $1.0 \mathrm{GPa}$ for 15 minutes. After exposure to $2.0 \mathrm{GPa}$ for the same time duration, the hatching rate had recovered to $33 \%$, but decreased to $8 \%$ following compression at $7.5 \mathrm{GPa}$. This contrasts with results using Fluorinert ${ }^{\mathrm{TM}}$ as the pressure-transmitting medium where $80-88 \%$ recovery was observed. The lower survival rate in water is accompanied by swelling of the eggs, indicating that liquid $\mathrm{H}_{2} \mathrm{O}$ close to the ice-VI crystallization pressure penetrated inside the eggs. This pressure exceeds the stability limit for proteins and other key biomolecules components within the embryos that could not be resuscitated. Rehydration takes several minutes and so was not completed for all samples compressed to higher pressures, prior to ice-VI formation, resulting in renewed survival. However $\mathrm{H}_{2} \mathrm{O}$ penetration inside the shell resulted in increased mortality.

Keywords: Artemia salina; cysts; high pressure tolerance; hatchability; osmotic pressure; ice VI phase

\section{Introduction}

It has been demonstrated that biological samples in a metabolically inactive state induced by dehydration or sporulation processes can withstand surprisingly high hydrostatic pressures, extending above several GPa, with a large fraction of the sampled population exhibiting full to partial recovery upon resuscitation after exposure to the high pressure treatment [1-15]. Such pressures in the GPa range greatly exceed those that are known to result in irreversible loss of biological functionality of proteins, cell membrane components, and other essential parts of the biomolecular machinery. We 
can conclude that the structure-function relationships among these biomolecules and biomolecular systems are protected from destruction in the metabolically arrested state by mechanical resistance of the external shell surrounding the eggs, cysts or seeds $[7,14$, 15]. These are typically formed from glycoprotein coatings along with complex sugars such as trehalose, and extracellular matrix (ECM) peptides that provide protection against chemical and environmental stressors such as dehydration, high salinity, low/high $\mathrm{pH}$ values [16], extreme temperatures and radiation damage [17, 18], and are developed via signalling pathways involving "heat shock" proteins [19]. The ECM provides a high degree of environmental protection, including resistance to compressive stress. Most previous investigations have focused on the tensile regime relevant to connective tissue such as cartilage, although a few have probed the responses to compressive resistance. We expect that the nanocomposite biomaterial that constitutes the ECM developed around Artemia cysts during the dehydration and dormant period. This should exhibit a high degree of mechanical resistance to rupture during compression, even extending into the GPa range of applied compressive stress-, but with the possibility of the Artemia cyst shells undergoing osmotic diffusion of $\mathrm{H}_{2} \mathrm{O}$ into the organisms to reverse their dehydrated state. In the first experiments to explore survivability of different types of organism, we carried out pressurization experiments of Artemia cysts using Fluorinert ${ }^{\mathrm{TM}}$ to apply pressure to the samples [2,3]. Here we further developed our study using water as the pressure-transmitting medium.

Artemia salina represents a type of plankton known as "brine shrimp" that are found in inland saltwater lakes. As the lake dries out, their eggs enter a metabolically inactive "cyst" state where they can survive for many years. The cysts then hatch into larvae called "Nauplii" after soaking for about $12 \mathrm{~h}$ in salt water. Artemia cysts have been shown to be highly tolerant of exposure to extremely high hydrostatic pressures. In 
previous experiments using Fluorinert ${ }^{\mathrm{TM}}$ as the high pressure medium, we observed the Nauplii hatching rate to remain as high as $88 \%$ following exposure to $7.5 \mathrm{GPa}$ for $36 \mathrm{~h}$, and $80 \%$ for $42 \mathrm{~h}[2,3]$. In our work reported here, we used water as the compression medium, and observe different results due to the relative permeability of the Nauplii shells to long-chain hydrocarbons $v s$ aqueous fluids. We note that carrying out similar experiments in brine might affect the hatching rate, and this remains to be investigated in future studies.

\section{Experimental Method}

Specimens for the present high pressure experiment were dehydrated eggs of Artemia which were kept in a low humidity container of about $20 \%$ for more than a week. Several tens of them were then sealed inside a Teflon ${ }^{\mathrm{TM}}$ capsule to conduct the high pressure experiments. The inner dimensions of the capsule used for experiments up to 5.5 GPa were $2.5 \mathrm{~mm}$ (diameter) and $3.0 \mathrm{~mm}$ (length), and $1.6 \mathrm{~mm}$ (diameter) and 1.8 $\mathrm{mm}$ (length) for 7.5 GPa experiments. We used pure water as the high-pressure medium. The capsule was inserted into the center of a pyrophillite cube that was then placed at the center of six WC anvils and compressed using a 250 -ton press. The press load was controlled to maintain constant pressure once the maximum pressure of the experiment was reached [1-3]. The pressure was increased to the experimental target value $(1.0,1.5,2.0,5.5,7.5 \mathrm{GPa})$ at $1.0 \mathrm{GPa} / \mathrm{min}$, where it was held constant for 15 minutes before decompression to ambient at the same rate. All experimental runs were performed at $300 \mathrm{~K}$. After decompression, the Artemia eggs were removed from the capsule and the morphology examined using optical microscopy. Hatching of Nauplii was observed after soaking the eggs in sea water and reanalysing under the microscope. 


\section{Experimental results}

\subsection{Hatching rate following high pressure exposure}

The observed hatching rate for control samples not exposed to high pressure was $75 \%$. This was lower than the hatching rates of $80-88 \%$ found in previous studies $[2,3]$, perhaps indicating either natural variability with the Artemia salina populations, or related to the fact that the eggs were stored in a refrigerator for a longer time. After exposure to $1.0 \mathrm{GPa}$ for 15 minutes, no eggs were observed to hatch following decompression to ambient conditions (Fig. 1). This result was confirmed by repeating the experiment 5 times. However, the hatching rate increased to $40 \%$ after exposure to $1.5 \mathrm{GPa}$, dropping to $33 \%$ after $2 \mathrm{GPa}$, then gradually decreased to $12 \%$ after $5.5 \mathrm{GPa}$, and $8 \%$ after $7.5 \mathrm{GPa}$. These survival rates are much lower than those that were maintained following 7.5 GPa compression in Fluorinert ${ }^{\mathrm{TM}}(80-88 \%)[2,3]$.

\subsection{Shapes of Artemia eggs exposed to different high pressures}

Artemia eggs in their dehydrated cyst state typically have a non-spherical shape with a dimpled exterior, like a deflated football. That same shape is preserved after soaking for $1 \mathrm{hr}$ in Fluorinert ${ }^{\mathrm{TM}}$, demonstrating that unlike pure water or brine, this fluid does not penetrate the external shell (Fig. 2). In Figure 3, we show images of the Artemia cysts following exposure to different pressures ranging up to $7.5 \mathrm{GPa}$. It is clear that the eggs exposed to $1.0 \mathrm{GPa}$, that do not hatch upon recovery to ambient conditions, are swollen and have adopted a spherical shape. That is only partly achieved for specimens exposed to $2.0 \mathrm{GPa}$, and the number decreases further with increasing pressure.

We examined the development of the spherical morphology as a function of time (10s, 5, 10 and 15 mins) while holding in water at $1.0 \mathrm{GPa}$ (Fig. 4). The time taken for all of the eggs to swell to a fully rehydrated state is on the order of several minutes, 
reflecting the kinetics of water diffusion throughout the sample chamber and pass through the egg shells. However, after 15 minutes exposure to the high pressure hydrostatic condition, all of the eggs had adopted a swollen spherical form. In contrast to this result, most of the eggs exposed to higher run pressures, following an experimental compression rate of $1.0 \mathrm{GPa} / \mathrm{min}$ to achieve the target pressure, exhibited a partially swollen state and did not achieve full spherical morphology (Figs. 3, 5). In Figure 6, we show an image of one of the Nauplii hatched from eggs that had been exposed to $5.5 \mathrm{GPa}$ for 1 hour. Although it appeared to hatch normally, we observed that its swimming speed was slower than specimens from control experiments.

\section{Discussion}

We first examine the effect of compression to different target pressures in the GPa range, using pure water $v s$ Fluorinert ${ }^{\mathrm{TM}}$ as a compression medium. The fact that soaking Artemia cysts in the fluorinated hydrocarbon does not result in any swelling indicates that this fluid medium does not penetrate the shell, unlike pure water or brine solutions (Fig. 2). The observation of $80-88 \%$ hatching rate following exposure to $7.5 \mathrm{GPa}$ in this compression medium $[2,3]$ suggests that the shell resists the external pressure conditions and the essential biomolecular systems contained inside the embryo remain undamaged. When the eggs are exposed to an aqueous pressurization medium, the cells become rehydrated, on a timescale extending from several seconds to minutes. At an experimental run pressure of $1.0 \mathrm{GPa}$, liquid $\mathrm{H}_{2} \mathrm{O}$ coexists with ice-VI crystals within the sample chamber, causing swelling of the cysts. However, this pressure exceeds the stability limit of the protein complexes, cell membranes and other elements of the delicate biomolecular machinery, which become no longer viable so that no hatching is observed. However, after ramping the pressure to $1.5 \mathrm{GPa}$ at $1.0 \mathrm{GPa} / \mathrm{min}$, the hatching 
rate was observed to increase again to $40 \%$. That result correlates with the observation that only partial penetration of the aqueous fluid can occur during compression to the target value, before formation of the immobile ice-VI phase. Inside the eggs compressed in $\mathrm{H}_{2} \mathrm{O}$, internal variations in hydration state and subsequent crystallization during compression will contribute to demise or damage to the Nauplii embryos. Once some water enters the cells during compression through the range in which liquid was present, this must contribute to the systematic lowering in hatching rate, and obvious mobility changes in re-hatched Nauplii, observed as the experimental hold pressure is increased to $7.5 \mathrm{GPa}$.

\section{Conclusion}

Our results using $\mathrm{H}_{2} \mathrm{O}$ as a compression medium to study hatching rates of Artemia eggs exposed to pressures up to $7.5 \mathrm{GPa}$ demonstrated a survival rate as low as $8 \%$, compared with previous results of $80-88 \%$ observed using Fluorinert ${ }^{\mathrm{TM}}$ as the pressuretransmitting medium. Also, the hatching rate dropped to $0 \%$ in duplicate experiments carried out at $1.0 \mathrm{GPa}$, close to the water-ice VI crystallization boundary. We suggest that $\mathrm{H}_{2} \mathrm{O}$ liquid present within the sample capsule at this pressure entered the eggs via osmotic driving forces, resulting in swelling and a spherical shape for the outer morphology. However, the internal protein complexes and other biomolecular machinery were damaged or destroyed by compression beyond their stability limits. During compression to higher target values at a rate of $1.0 \mathrm{GPa} / \mathrm{min}$, water only partially penetrated the egg envelopes, before the medium formed the ice-VI phase, and so the embryos suffered minimal compression-induced damage. The fact that eggs exposed to $7.5 \mathrm{GPa}$ in Fluorinert ${ }^{\mathrm{TM}}$ remain viable demonstrates the intrinsic compression resistance of the outer envelope. The fact that complex multicellular 
organisms such as Artemia can withstand high pressures on the order of GPa has implications for industrial applications of food processing using "Pascalization" high pressure techniques.

\section{References}

[1] F. Ono, M. Saigusa, T. Uozumi, Y. Matsushima, H. Ikeda, N. L. Saini, M. Yamashita: J. Phys. Chem. Sol. 69 , 2297 (2008).

[2] F. Ono, K. Minami, M. Saigusa, Y. Matsushima, Y. Mori, K. Takarabe, N. L. Saini, M. Yamashita: J. Phys. Chem. Solids, 71, 1127 (2010).

[3] K. Minami, F. Ono, Y. Mori, K. Takarabe, M. Saigusa, Y. Matsushima, N. L. Saini, M. Yamashita: J. Phys. Conf. Series, 215, 12164 (2010).

[4] N. Nishihira, A. Shindou, M. Saigusa, F. Ono, Y. Matsushima, Y. Mori, K.

Takarabe, N. L. Saini, M. Yamashita: J. Phys. Chem. Solids, 71, 1123 (2010).

[5] F. Ono, Y. Mori, K. Takarabe, N. Nishihira, A. Shindo, M. Saigusa, Y. Matsushima, N. L. Saini, M. Yamashita: J. Phys. Conf. Series, 215, 12165 (2010).

[6] N. Nishihira, T. Iwasaki, R. Shinpou, A. Hara, F. Ono, Y. Hada, Y. Mori, K.

Takarabe, M. Saigusa, Y. Matsushima, N. L. Saini, M. Yamashita: J. Appl. Phys., 111, 112619 (2012).

[7] F. Ono, Y. Mori, M. Sougawa, K. Takarabe, Y. Hada, N. Nishihira, H. Motose, M. Saigusa, Y. Matsushima, D. Yamazaki, E. Ito, N. L. Saini: J. Phys. Conf. Series, 377, 12053 (2012).

[8] Y. Mori, S. Yokota, F. Ono: J. Phys. Conf. Ser., 377, 12055 (2012).

[9] M. Shibata, M. Torigoe, Y. Matsumoto, M. Yamamoto, N. Takizawa, Y. Hada, Y. Mori, K. Takarabe, F. Ono: J. Phys. Conf. Series, 500, 102004 (2014). 
[10] F. Ono, N. Nishihira, Y. Hada, Y. Mori, K. Takarabe, M. Saigusa, Y. Matsushima,

D. Yamazaki, E. Ito: J. Phys. Chem. Solids, 84, 57 (2015).

[11] S. Tanaka, H. Motose, F. Ono, M. Yamashita, M. Saigusa: Space Utiliz. Res., 27, 171 (2011). (in Japanese)

[12] R. Hazael, F. Meersman, F. Ono, P. F. McMillan: Life, 6, 34 (2016).

[13] F. Ono, Y. Mori, K. Takarabe, A. Fujii, M. Saigusa, Y. Matsushima, D. Yamazaki, E. Ito, S. Galas, N. L. Saini: Cogent Physics, 3, 1167575 (2016).

[14] F. Ono: High Pressure Bioscience, Subcellular Biochemistry 72 (K. Akasaka, H. Matsuki eds., Springer, 2015) Chap. 22, pp. 443-466.

[15] F. Ono, N. Nishihira, M. Sougawa, Y. Hada, Y. Mori, K. Takarabe, M. Saigusa, Y. Matsushima, D. Yamazaki, E. Ito, N. L. Saini: High Pres. Res., 33, 362 (2013).

[16] K. Takai, D. P. Moser, T. C. Onstott, N. Spoelstra, S. M. Pfiffner, A. Dohnalkova, J. K. Fredrickson, Int. J. Syst. Evol. Microbiol. 51, 1245 (2001).

[17] D. D. Horikawa, T. Sakashita, C. Katagiri, M. Watanabe, T. Kikawada, Y.

Nakahara, N. Hamada, S. Wada, T. Funayama, S. Higashi, Y. Kobayashi, T. Okuda, M. Kuwabara, Int. J. Radiat Biol. 82, 843 (2006).

[18] T. Hashimoto, D. D. Horikawa, Y. Saito, H. Kuwahara, H. K. Hata, T. Shin-I, Y. Minakuchi, K. Ohishi, A. Motoyama, T. Aizu, A. Enomoto, K. Kondo, S. Tanaka, Y. Hara, S. Koshikawa, H. Sagara, T. Miura, S. Yokabora, K. Miyagawa, Y. Suzuki, T. Kubo, M. Oyama, Y. Kohara, A. Fujiyama, K. Arakawa, T. Katayama, A. Toyoda, T. Kunieda, Nature Commun. 712808 (2016).

[19] D. Whitley, S. P. Goldberg, W. D. Jordan: J. Bascular Surgely, 29, 748 (1999). 


\section{Figure captions}

Figure 1. Observed hatching rate of Artemia eggs exposed to high pressures for 15 minutes using pure water as the pressure medium. The positions of ice VI crystallization and ice VI-VII transition boundaries at $300 \mathrm{~K}$ are indicated. The data are compared with previous results using Fluorinert ${ }^{\mathrm{TM}}$ as the pressure-transmitting medium.

Figure 2. The effects of soaking unpressurized Artemia cysts in different media. (a) After soaking in Fluorinert ${ }^{\mathrm{TM}}$ for $60 \mathrm{~min}$; (b) After soaking for $60 \mathrm{~min}$ in pure water; (c) After soaking for $60 \mathrm{~min}$ in sea water.

Figure 3. Artemia eggs photographed in the Teflon capsule. (a) before exposure to high pressure, (b) after exposure to $1.0 \mathrm{GPa}$ for 15 minutes, (c) after exposure to $2.0 \mathrm{GPa}$, (d) after 5.5 GPa and (e) after 7.5 GPa. The spherical expanded morphology of the cysts in (b) is clearly seen, in comparison with the other results.

Figure 4. Artemia cysts in their Teflon capsule following exposure to $1.0 \mathrm{GPa}$ in pure $\mathrm{H}_{2} \mathrm{O}$ for different times. (a) $10 \mathrm{~s}$, (b) 5 mins, (c) $10 \mathrm{~min}$ (d) 15 min. Close up view of Artemia cysts following 1.0 GPa exposure to $\mathrm{H}_{2} \mathrm{O}$ : (e) $10 \mathrm{~s}$, (f) $10 \mathrm{~min}$, (g) $15 \mathrm{~min}$.

Figure 5. Image of an Artemia cyst exposed to $2 \mathrm{GPa}$ in pure water for 15 minutes.

Figure 6. One of the Nauplii hatched from eggs exposed to $5.5 \mathrm{GPa}$ for 1 hour using pure water as the high pressure medium. 
Figure 1.

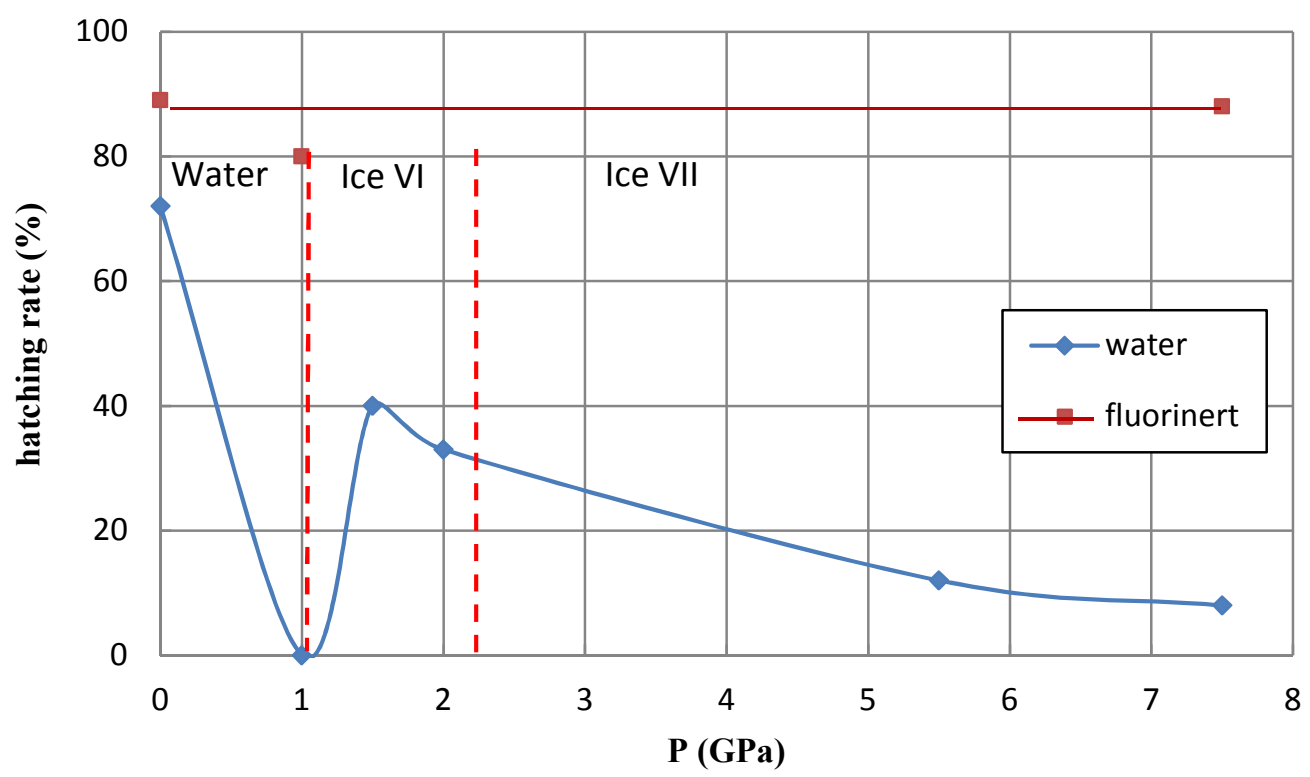


Figure 2.
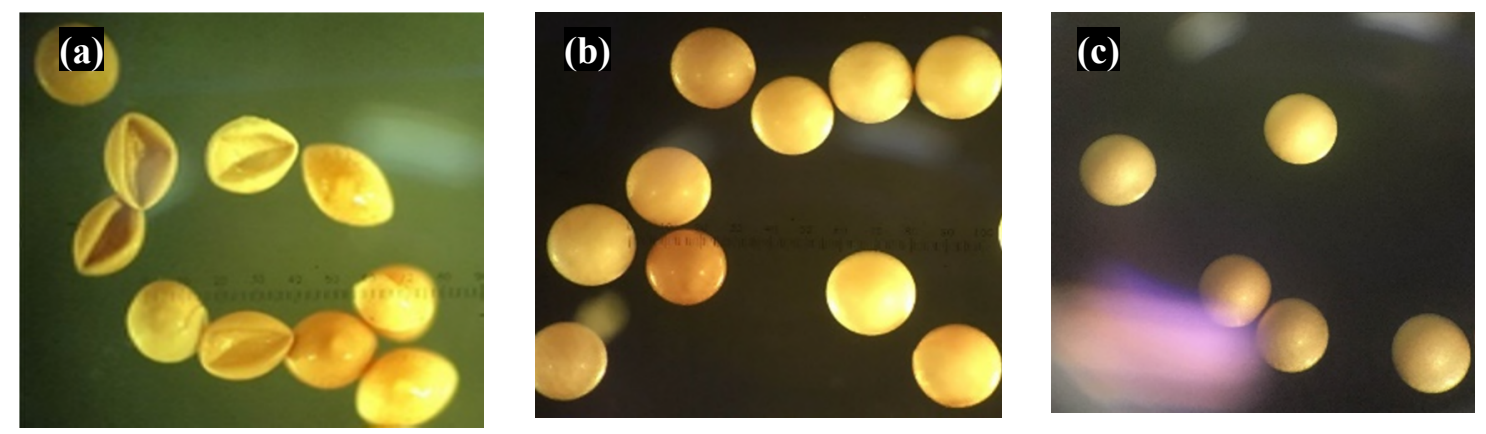
Figure 3.

(a)

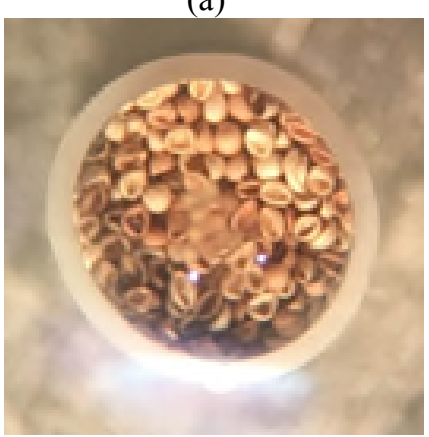

(c)

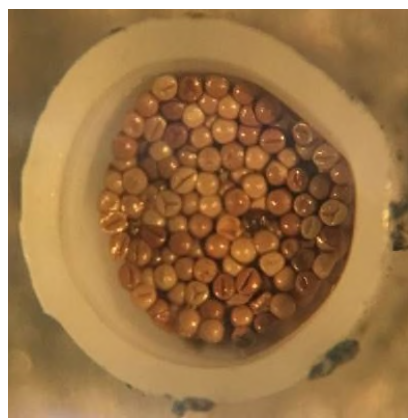

(b)

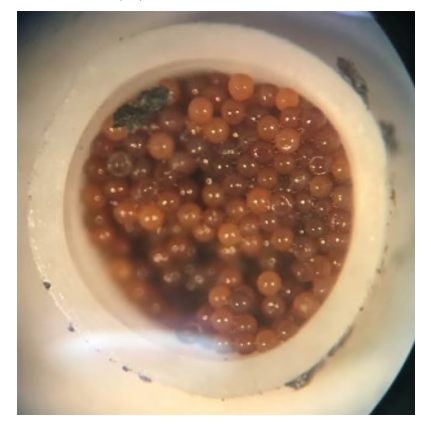

(e)

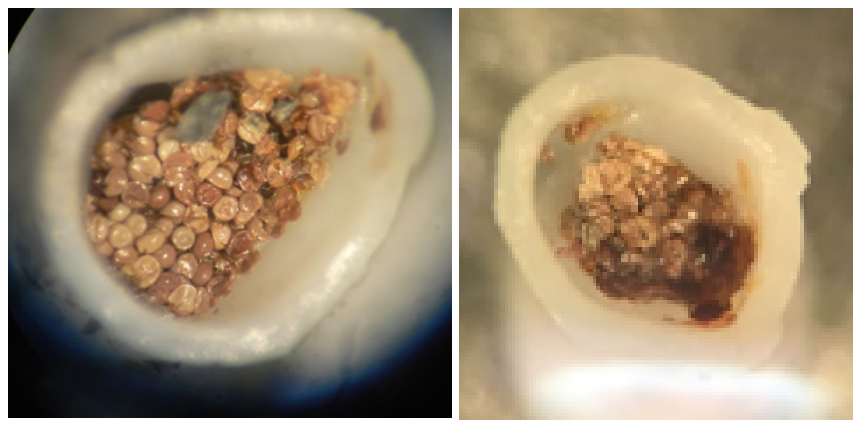


Figure 4.
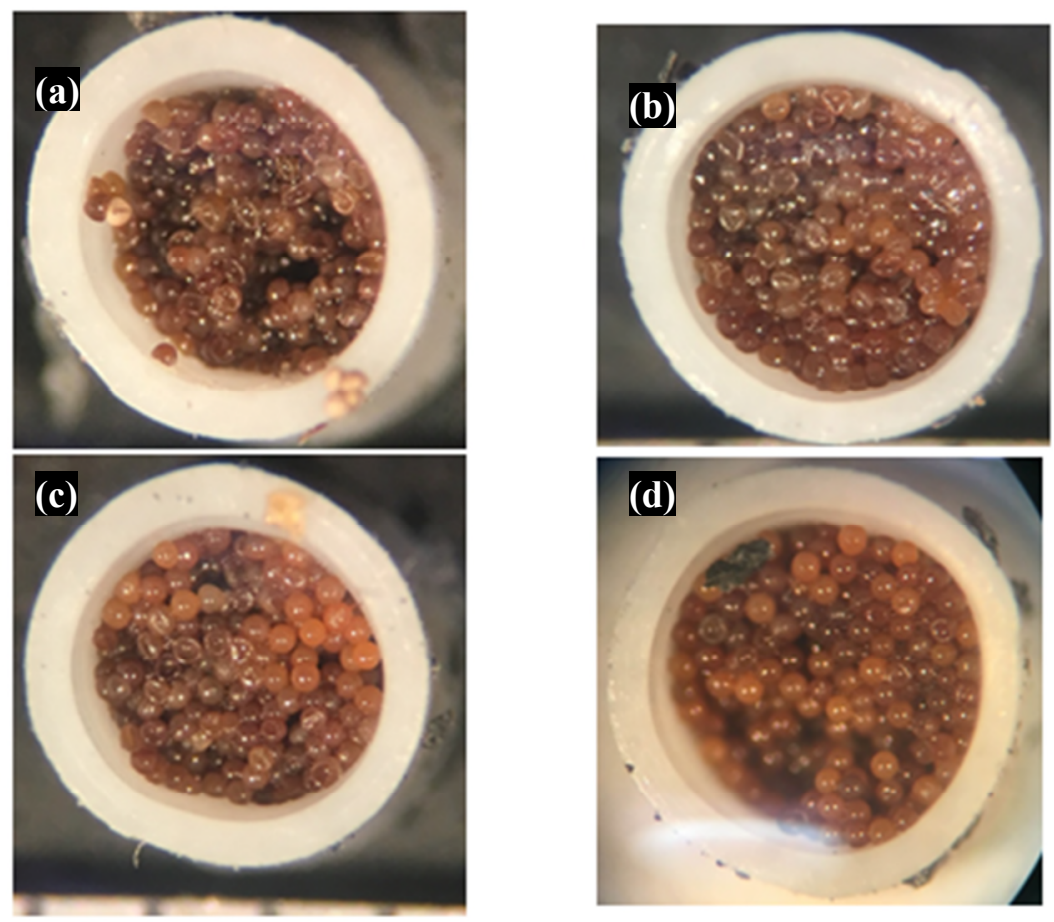
Figure 4.

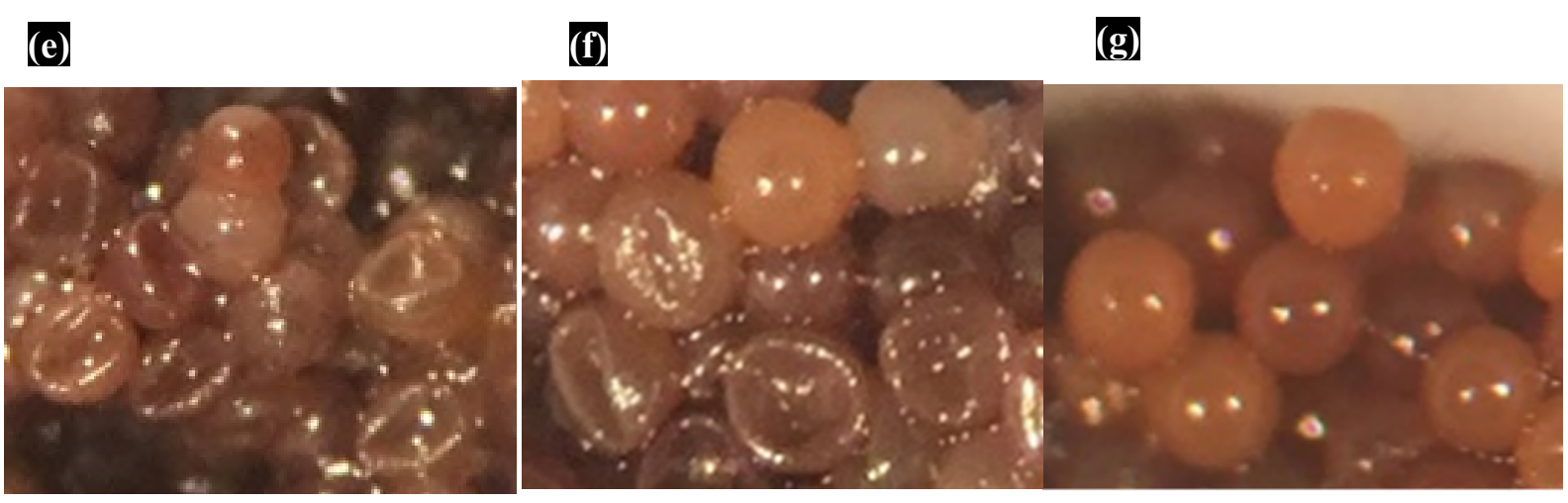

Figure 5.

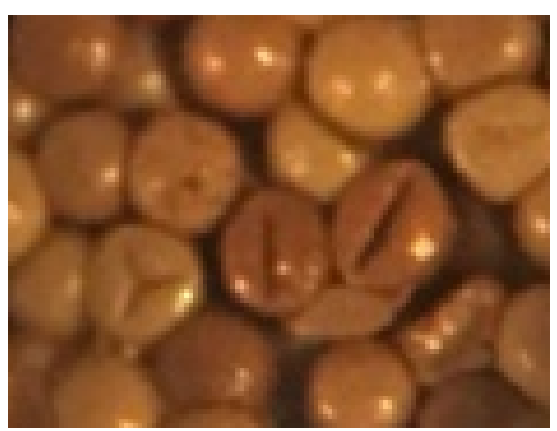


Figure 6.

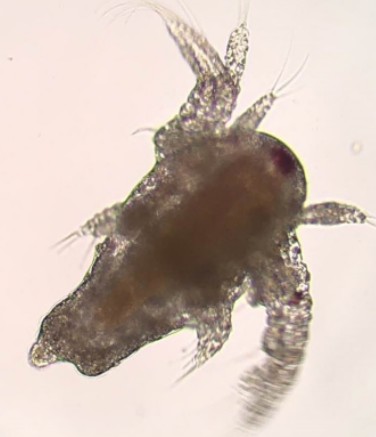

